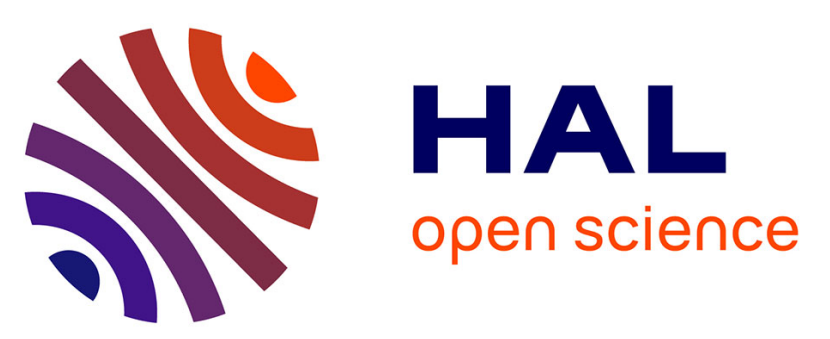

\title{
Using Generic Upper-Body Movement Strategies in a Free Walking Setting to Detect Gait Initiation Intention in a Lower-Limb Exoskeleton
}

Omar Mounir Alaoui, Fabien Expert, Guillaume Morel, Nathanael Jarrasse

\section{- To cite this version:}

Omar Mounir Alaoui, Fabien Expert, Guillaume Morel, Nathanael Jarrasse. Using Generic UpperBody Movement Strategies in a Free Walking Setting to Detect Gait Initiation Intention in a LowerLimb Exoskeleton. IEEE Transactions on Medical Robotics and Bionics, 2020, 2 (2), pp.236-247. 10.1109/TMRB.2020.2982004 . hal-02541938

\section{HAL Id: hal-02541938 \\ https://hal.science/hal-02541938}

Submitted on 14 Apr 2020

HAL is a multi-disciplinary open access archive for the deposit and dissemination of scientific research documents, whether they are published or not. The documents may come from teaching and research institutions in France or abroad, or from public or private research centers.
L'archive ouverte pluridisciplinaire HAL, est destinée au dépôt et à la diffusion de documents scientifiques de niveau recherche, publiés ou non, émanant des établissements d'enseignement et de recherche français ou étrangers, des laboratoires publics ou privés. 


\title{
Using Generic Upper-Body Movement Strategies in a Free Walking Setting to Detect Gait Initiation Intention in a Lower-Limb Exoskeleton
}

\author{
Omar Mounir Alaoui, Fabien Expert, Guillaume Morel, Nathanaël Jarrassé
}

\begin{abstract}
In recent years, lower-limb exoskeletons have been marketed to become a possible alternative to wheelchairs for people with walking impairments or paralysis. However, most assistive exoskeletons rely on constraining control strategies based on remote controls or torso tilting events. One approach to build more intuitive control interfaces would be to exploit knowledge on human motor control, and the coordination between upper and lower limb movements during gait events, such as the anticipatory postural adjustments that precede gait initiation.

In this study, it was hypothesized that generic trunk and arm movements preceding gait in a free walking setting in able-bodied users can still be retrieved while wearing an assistive lower-limb exoskeleton that constrains their leg movements. This was evaluated on a group of eight unimpaired participants, and analysis of upper-body wearable IMU signals showed similarity of postural adjustments between the free and exoskeleton-constrained settings. Additionally, a classification architecture showed that the walking state of the robot can be correctly triggered based on free-walking data gathered from all participants with an accuracy rate of $95 \%$. This suggests that interlimb coordination still exists in a constrained setting, and could pave the road towards the elaboration of more natural controls for assistive lower-limb exoskeletons.
\end{abstract}

\section{INTRODUCTION}

Recent developments in the fields of robotics and mechatronics have made it possible to design lower-limb assistive devices, such as exoskeletons and active orthoses, that could be used for physical augmentation or as an alternative to wheelchairs for physically impaired individuals and Spinal Cord Injury (SCI) patients [1], [2]. Although such devices still face important challenges - many of which revolve around actuation, cost, weight, and human-machine interfaces [3]-[5] - a certain number of companies have been able to market and industrialize their own exoskeletons, with a focus on assisting SCI patients, or helping with the rehabilitation of stroke patients. However, most of them still rely on constraining control strategies that fail with providing the users with natural and intuitive interfaces.

The ReWalk exoskeleton (ReWalk Robotics Ltd., Yokneam, Israël) makes use of a wrist-pad controller to control the state of the robot, and a tilt sensor placed on the torso to trigger its movements; but crutches are necessary to stabilize the robot, which greatly impedes free arm movements [6]. The

O.M. Alaoui, N. Jarrassé, and G. Morel are with the Institute for Intelligent Systems and Robotics, Sorbonne Université, Paris 75006, France. (e-mail: alaoui@isir.upmc.fr)

O.M. Alaoui and F. Expert are with Wandercraft, Paris 75004, France. (email: fabien.expert@wandercraft.eu)
Rex exoskeleton (Rex Bionics Ltd, Auckland, New Zealand), while being able to stabilize itself, still necessitates the use of a manual joystick to be controlled [7]. In 2012, the french start-up company Wandercraft (Paris, France) has taken up the challenge to develop a dynamically stable lower-limb exoskeleton for paraplegic patients [8], [9]. Their robot, named Atalante (Fig. 1), does not rely on crutches for stabilization, but still makes use of remote buttons to switch between its different states, and an Inertial Measurement Unit (IMU) to trigger specific movements based on upper-trunk kinematics.

In a context where patients wish for such assistive devices to become physical extensions of their own bodies, it is necessary to develop control interfaces that are not only robust, efficient, and safe, but feel natural and intuitive as well [10], [11]. Numerous studies have focused on the use of electrophysiological signals (such as electromyograms EMGs - or electroencephalograms - ECGs) to decode human motor intentions and provide an external device with input control signals [12]-[14]. But the variability of these signals, and the lack of robustness of the decoding schemes make it difficult to apply such methods in an uncontrolled setting [15], [16].

A different approach consists in analyzing body movements that can be predictive of specific motor intentions, or to rely on kinematic synergies between different parts of the body. The common paradigm currently adopted by the ReWalk or Atalante exoskeletons is based on simple threshold-based approaches that rely on the detection of predefined movements that need to be learned, such as a high amplitude leaning motion, or on simple button-mapped commands. However, by making use of machine learning techniques, it can be possible to reverse the adaptation paradigm, and build more natural control strategies where supervised algorithms can learn from naturally exhibited human movements, instead of having the users adapt to predefined control patterns. Such techniques have already been widely used for IMU-based human activity recognition [17]-[20], but they can also be applied to classify shorter transitioning predictive movements.

For example, the turning intention of healthy participants during gait can be predicted based on anticipatory movements of the head, eyes, trunk, or shoulders [21]-[23]. Similarly, stereotypical patterns of body movements are involved during gait initiation as a planned compensation to the perturbation introduced by the heel-off of the swing leg. This set of anticipatory postural adjustments (APAs) shifts the center of mass by accelerating it forwards and laterally towards the 


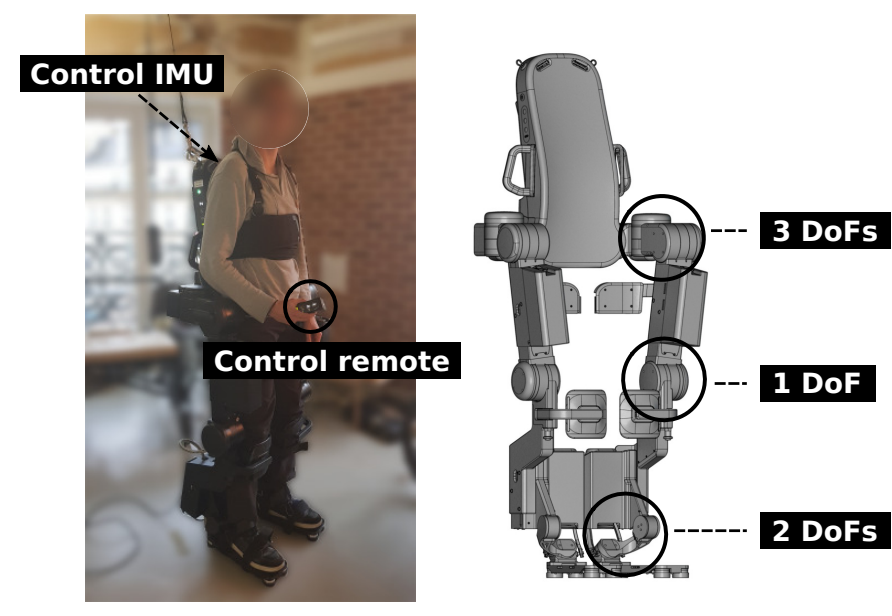

Fig. 1. The 12-DoF lower-limb assistive exoskeleton Atalante developed by the French company Wandercraft. Atalante is mainly aimed at being used by paraplegic patients who can still use their upper-body, and as a rehabilitation device for stroke patients.

stance leg, and has been mainly investigated through force sensors underneath the feet, optical motion trackers, and IMUs placed on different upper and lower body parts [24], [25]. More recently, research teams have focused on the automatic detection of APAs based on IMU signals from both the upper and lower body, using various learning approaches [26] [28]. However, these investigations were limited to unimpaired participants walking freely in an unconstrained environment.

This study focuses on gait initiation, and investigates whether its precursor patterns can still be retrieved through upper body kinematics when unimpaired participants are constrained by an assistive lower-limb exoskeletal structure that prevents free leg movements. It was hypothesized that generic upper limb movements during anticipatory postural adjustments preceding the first heel-off event in a free-walking setting are preserved in the exoskeleton-constrained environment, and can therefore be used to predict the user's intention to initiate gait while in the robot. A comparison of IMU signals from the upper body during gait initiation between both the free (out of the robot) and constrained (in the robot) settings was conducted on a group of healthy unimpaired participants to validate this hypothesis. Additionally, data from the free walking setting was used as a training set to build a linear discriminant analysis (LDA) classification architecture that can automatically detect gait initiation intention. This classifier was first tested offline for validation on the free walking data and to assess intra and inter-subject variability. It was then implemented online as part of the exoskeleton's state machine. This allowed to experimentally evaluate the possibility of building natural and intuitive controls based on training data gathered outside of the robot from the free condition. By additionally recording typical everyday movements that one can perform while standing still in a lower-limb assistive exoskeleton, the robustness of such a classifier against false walk-triggering positives was also investigated. Correctly triggering the walking state of Atalante would confirm that upper body movements that anticipate gait initiation are possibly preserved between the free and constrained settings, and can be used to elaborate more natural and robust control strategies for exoskeletal assistive devices based on classification techniques, with limited to no false positive detection.

\section{MATERIALS AND METHODS}

\section{A. Experimental setup}

The study consisted of two experiments. In the first experiment, there were two conditions in which ten participants (7 men and 3 women) took part, as approved by the Ethical Committee on Research of the Paris Descartes University (IRB number 00012019-47) according to the standards of the Declaration of Helsinki. The participants were aged 29.9 \pm 4.3 years old, with an average weight of $67.5 \pm 13.32 \mathrm{~kg}$ and an average height of $174.7 \pm 9.70 \mathrm{~cm}$ (mean \pm SD). They had no physical or cognitive disabilities affecting gait, and were not aware of the study's focus on gait initiation. The first condition was conducted in a Free Setting, with participants outside of the Atalante exoskeleton (FS condition), and the second one was conducted in a Constrained Setting, with participants in the robot (CS condition). A second experiment was conducted in the exoskeleton to assess the robustness of the classification algorithm to False Positives (FP experiment). For both experiments, participants were equipped with three inertial measurement units (Next Generation IMUs, x-io Technologies) placed on both arms and the back (Fig. 2-A).

The inertial units were placed so that their axes were aligned with the global frame formed by the Medio-Lateral (ML) and Antero-Posterior (AP) planes, as defined in Fig. 2-A. The (Back-Front) and (Left-Right) axes in Fig. 2-A respectively represent the AP and ML axes. The vertical axis is defined by the intersection of the ML and AP planes, and points up. In all following sections, AP accelerations will refer to accelerations along the AP axis, and ML accelerations will refer to accelerations along the ML axis. Similarly, AP angular velocities will refer to angular velocities contained in the AP plane around the ML axis, and ML angular velocities will refer to angular velocities contained in the ML plane around the AP axis.

Accelerometer and gyroscope signals from all three sensors were sent wirelessly to a computer at $400 \mathrm{~Hz}$ through a Wi-Fi receiver using UDP protocol. In the free setting, participants were asked to remove their shoes, and were additionally equipped with a force sensitive resistor (FSR) placed underneath the right heel. The FSR was connected to the back IMU through an analog channel, and the signal was transmitted at $10 \mathrm{~Hz}$. It was used to segment the walking data into left and right steps, and facilitate the training data labeling process.

1) First Experiment: Free Setting (FS) condition: Instructions were given to the participants after being equipped with the IMUs and the FSR. The first condition consisted of 20 trials, in which the participants performed a straight walk of approximately $4 \mathrm{~m}$ at their preferred pace. At the beginning of each trial, they were asked to stand still in a neutral position behind a specified line on the ground, with their arms alongside their body, and their head straight and gazing forwards (Fig. 2-A).

The IMUs were initialized to set the reference frames at the neutral position, after which the recording began. An audio 

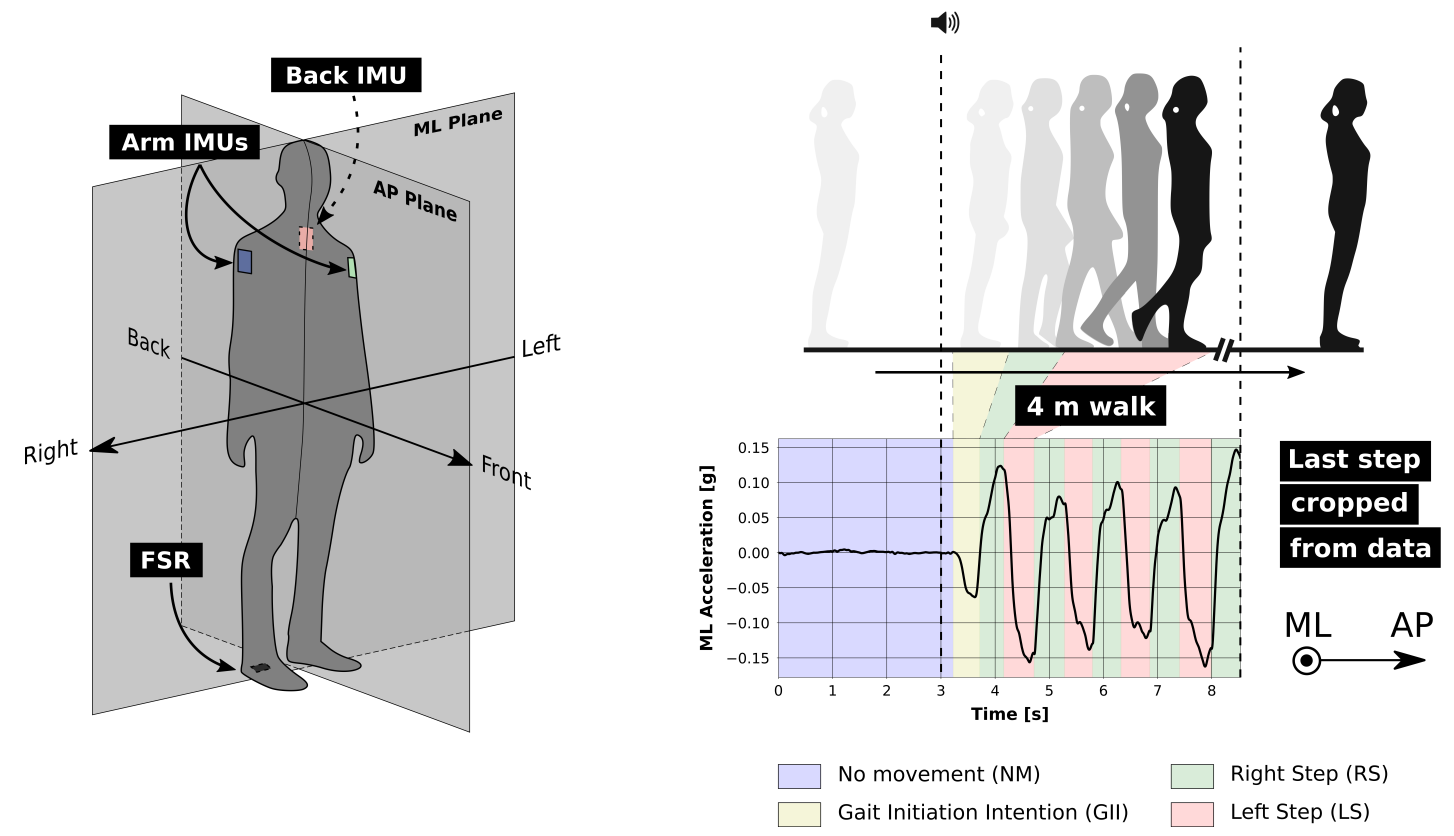

Fig. 2. (A) Illustration of a subject during the FS condition. The IMUs were aligned so that the $x$-axis was pointing upwards (vertical axis), the $y$-axis was pointing towards the right (medio-lateral axis), and the $z$-axis was pointing forwards (vertical axis). (B) Illustration of one trial from the FS condition. The signal represents labeled ML acceleration data from the back IMU in [rad/s] after the last step was removed. Blue indicates the NM class, yellow indicates the GII class, green indicates the RS class, and red indicates the LS class.

cue was emitted after $3 \mathrm{~s}$, indicating that the participants could start walking. To avoid any startle effects due to the emitted beep, participants were asked to start walking whenever they wanted to after hearing the sound cue. They were also asked to use their right leg as the first swing leg. At the end of the $4 \mathrm{~m}$ walk, participants stood still in their neutral position for approximately $2 \mathrm{~s}$, after which they were instructed to go back to the starting position, and wait for the beginning of the following trial (Fig. 2-B).

\section{2) First Experiment: Constrained Setting (CS) condition:}

In the second condition, 20 trials were conducted in which the participants were first equipped with all three IMUs, then installed in the exoskeleton. The Atalante exoskeleton (Fig. 1) is a full-joint assistive device designed by the French company Wandercraft. It has 12 actuated degrees of freedom (3 at each hip, 1 at each knee, and 2 at each ankle), and is dynamically stable - meaning that it does not rely on crutches for stabilization. Only two of the participants had never been in the exoskeleton before. The robot was put into its standard standstill position, and participants were instructed to stay motionless with their arms alongside their body, and their heads straight and gazing forwards. Similarly to the FS condition, the inertial devices were initialized at the beginning of each trial to set the reference frames, and an audio cue was emitted $3 \mathrm{~s}$ after the beginning of the recording to indicate the beginning of the walk. Subjects were asked to perform any upper body movements they thought would initiate the robot's gait, as if they wanted to start walking using their right leg. Since two participants did not correctly follow this rule, their results were discarded from the CS condition analysis, which only included eight participants. In 15 out of 20 trials, an online classifier based on linear discriminant analysis (LDA, see II-C) was used to detect gait intention using one of three training sets:

(a) same subject data from the FS condition (intraclassification);

(b) other participants data from the FS condition (interclassification);

(c) all participants data from the FS condition (global classification).

If gait intention was correctly detected, the walking state of the robot was triggered, in which case the participants were asked to stop the robot using its remote control after a few steps. In the remaining 5 trials, (d) the exoskeleton walk was not triggered at all. These four conditions were presented in completely random order across all 20 trials.

3) Second Experiment: False Positives (FP) experiment: In the second experiment, eight of the ten participants were equipped with all three IMUs, and installed in the Atalante exoskeleton. The robot was put into its standard standstill position, and similarly to the CS condition, participants were asked to stay motionless with their arms alongside their body, and their heads straight and gazing forwards. They were then instructed to wait for an audio cue, after which they were asked to perform a set of typical everyday movements: handshakes, covering the mouth while coughing, and reaching an object at different levels (below the waist, at torso level, and over the head). Each movement was alternatively performed with each arm, and repeated for 4 trials. Data recordings from this experiment were analyzed offline. 


\section{B. Data processing and analysis}

Data from the FS condition were processed offline. Accelerometer and gyroscope signals were low-pass filtered using a second-order Butterworth filter with cutoff frequency of 3 $\mathrm{Hz}$, as used in [27]. Signals were also offset based on their average over the first second of recording, during which the participants were standing still in a neutral position. Similar filtering and offset were performed online for data during the CS condition. Trials where indications were not correctly followed, or which signal waveforms differed significantly from the average ones were considered outliers, and discarded. A total of 170 trials were retained for the subsequent analysis.

\section{Data classification}

1) Linear Discriminant Analysis: Data classification was performed using Linear Discriminant Analysis (LDA) [29]. LDA is a supervised learning algorithm which separates labeled data in an input feature-space using optimally-defined hyperplanes, by maximizing between-class covariance and minimizing within-class covariance. New data points in the feature-space are classified according to the highest signed distance to the class hyperplanes, as generated by training data. LDA can also be used as a dimensionality reduction technique, by projecting data onto subspaces of dimensions at most equal to the number of classes minus one. This was used in the scope of this work for visualization purposes.

2) Training Data labeling: Accelerometer and gyroscope signals from the FS condition were segmented into four different classes: No Movement (NM), Gait Initiation Intention (GII), Right Step (RS), and Left Step (LS). Heel events of the right foot were derived from the FSR data for step labeling. The FSR data during the first three seconds of recording were averaged to set a standing still baseline for each trial. Heel-strike events were then set when the FSR signal was higher than the baseline plus one standard deviation, and heeloff events were set when the FSR signal was lower than the baseline minus one standard deviation. RS was defined as the class covering signal portions going from heel-off to heel-strike, and LS was defined as the class covering signal portions going from heel-strike to heel-off. Data following the last heel-strike event were discarded, as last step dynamics differed from steady-pace walking. The first detected upperbody movements on the IMU data were used to determine the onset time $t_{\text {onset }}$ for the GII class. Detection was achieved by setting movement thresholds $T_{+, s i g}$ and $T_{-, s i g}$ for each accelerometer and gyroscope signal sig $\in\left\{\ddot{x}, \ddot{y}, \ddot{z}, \omega_{x}, \omega_{y}, \omega_{z}\right\}$ as:

$$
\begin{aligned}
& T_{+, s i g}=m_{s i g}+10 \times s_{s i g} \\
& T_{-, s i g}=m_{s i g}-10 \times s_{s i g}
\end{aligned}
$$

where $m_{\text {sig }}$ (resp. $s_{\text {sig }}$ ) is the average (resp. standard deviation) of each signal over the $1.5 \mathrm{~s}$ preceding the audio cue. For each signal, $t_{\text {min,sig }}$ was then defined as the earliest time such that sig $\notin\left[T_{-, \text {sig }}, T_{+, \text {sig }}\right]$, and $t_{\text {onset }}$ was defined as:

$$
t_{\text {onset }}=\min _{\text {sig }} t_{\text {min }, \text { sig }}
$$

The first heel-off was used as the end of the GII class. The remaining portions of signals, spanning from the beginning of each trial up to the GII onset, were labeled as NM. A final step consisted in manually adjusting the class onsets if outputs from the above steps were incorrect. Fig. 2 shows an example of labeled accelerometer data.

For the second experiment, an additional class based on miscellaneous movements (MM) was added to evaluate the classification robustness. The MM class was constructed based on recordings of typical everyday movements from the FP experiment.

3) Classifiers construction: Once accelerometer and gyroscope data from all trials and participants were correctly labeled, they were used as training sets to construct LDA classifiers. Sliding windows with a $25 \%$ overlap were used to divide the sensor signals into $500 \mathrm{~ms}$ time segments. Sliding windows are commonly used for activity classification [30] [20]. The choice of window length was motivated by reported values for Anticipatory Postural Adjustments (APAs) duration in [24] [25] (350 - $550 \mathrm{~ms})$. The overlap value was chosen to enhance the classifier reaction time. Each window was associated with a given class if at least half of its signal content was labeled as so, based on the labeling step. A set of optimally relevant features were then extracted from each segment to compute labeled feature-space data points. They were selected based on previous research [30] [20]. In order to reduce the computational cost for features extraction in realtime, and avoid overfitting, further analyses were conducted to discard features which were not discriminative enough for the classification task. In particular, a single-factor ANOVA between labels and features was conducted to only select features with high variance across the different classes. This allowed to define a subset of 11 features, which were computed from both the time and frequency domains: mean, standard deviation ( $\mathrm{sd}$ ), minimum ( $\min )$, maximum ( $\max$ ), root mean square (rms); spectral energy, and the five highest amplitudes of the frequency components in the frequency-domain. Since all features were extracted from each of the 18 IMU signals, this resulted in a final 198-dimensional feature space (see Table I).

TABLE I

SET OF FEATURES USED IN CLASSIFIERS CONSTRUCTION

\begin{tabular}{llc}
\hline & Feature & Number of dimensions \\
\hline Time-domain features & mean & 18 \\
& $\mathrm{sd}$ & 18 \\
& $\min$ & 18 \\
& $\max$ & 18 \\
& $\mathrm{rms}$ & 18 \\
\hline Frequency-domain features & spectral energy & 18 \\
& highest amplitudes & 90 \\
\hline Total & 7 features & 198 \\
\hline
\end{tabular}

4) Training and testing data:

a) Free Setting (FS): condition

Trial-based cross-validation schemes were used to assess data classification for the FS condition. In a first setting, subject-specific data were classified according to a leave-one- 
out rule: training sets were comprised of all but one trial from the same subject, which was then used for testing. (intrasubject classification). This was done over all trials, and the overall result was taken as the total average of all classification rates on the testing data. In a second setting, classification was assessed across participants, by leaving one subject's trials from the training set, and using them as a testing set (intersubject classification). The overall result was taken as the total average from testing the classifier on each of the leftout subject's trials independently.

Additionally, the Inter-class Distance Nearest Neighbor (IDNN) metric as proposed in [31] was used to compute distances in the original 198-dimensional feature space formed by the training data for each subject. For each cluster of points, it measures the product of its Mahalanobis distances with the other clusters in both directions, and normalizes it by their sum. The IDNN for a given cluster is then equal to the lowest value computed across all the other ones, and represents the distance to its nearest neighbor in a variance-normalized space. A low IDNN value for a given class indicates a larger chance of confusion with its nearest neighbor. Equal IDNN values for two different classes indicate that they are necessarily closest to each other in the sense of the Mahalanobis distance. Details on the computation of the IDNN are given in the appendix.

b) Constrained Setting (CS) condition: In the CS condition, three classifiers were constructed for each subject based on the training data from the FS condition: (a) one subject-specific classifier based on the subject's trials (intraclassification), (b) one classifier based on the other participants' trials (inter-classification), (c) and an additional global classifier including all participants data, which was common to all participants (global classification). These classifiers were tested online during the CS condition.

c) False Positives (FP) experiment: Data sets recorded during the FP experiment were first tested offline using the global classifier to test for the occurrence of false GII positives when performing everyday movements. Half of the data were then included with all the participants data from the FS condition to construct an enriched 5-class global classifier, which was tested offline:

- on the remaining half of the FP experiment data;

- and on the trials from the CS condition corresponding to the global classifier.

\section{RESULTS}

\section{A. Upper-body kinematics indicating Gait Initiation Intention (GII)}

Fig. 3 shows the average accelerations and angular velocities for all three IMUs in the medio-lateral (ML) and anteroposterior (AP) planes over all participants from the Free Setting (FS) condition. Signals are represented from $0.5 \mathrm{~s}$ before the movement onset up to the first heel strike event. The green mark indicates the first heel-off event, and the red one indicates the first heel strike event. The average duration ( \pm SD) between the movement onset and the heel-off is 546 $\mathrm{ms}$ ( $\pm 132 \mathrm{~ms}$ ) for this condition.
Fig. 4 shows the average accelerations and angular velocities over the eight participants from the Constrained Setting (CS) condition for all three IMUs in the ML and AP planes. The signals are represented from $0.5 \mathrm{~s}$ before the movement onset, up to the first zero-crossing of the angular velocity around the vertical axis.

In both figures, the shaded areas represent the standard deviations for the different signals. For waveform comparison purposes, and to get rid of amplitude variability effects, individual trial results have been standardized and represented as functions of time advancement (as a percentage value from 0 to 100$)$.

Signals from the CS condition in Fig. 4 have been further filtered with a low-pass Butterworth (2nd order, cutoff 0.5 $\mathrm{Hz}$ ) to get rid of extraneous noise, and focus on global signal evolution in time. This was only done to improve readability of the figure, but was not part of the data processing that occurred during the experiment. However, averaging and filtering techniques had a dampening effect on the representation of the signals in Fig. 4. Fig. 5 shows an example of a typical AP acceleration profile exhibited by one subject on the right arm IMU during the CS condition without additional processing, as was used by the classifiers. It shows that a short acceleration phase immediately follows the movement onset, before the acceleration direction changes.

Average peak values for the acceleration phases in the ML and AP planes for both conditions are reported in Table II, and they are indicated for the FS condition on Fig. 3. Maximum amplitude during precursor movements for acceleration signals is always reached before the heel-off event, except for the AP acceleration from the left arm, which is slightly delayed and happens after heel-off. Additionally, average peak values of gyroscope signals from the FS condition, and average peak gyroscope values from the CS signals as represented on Fig. 4 are also reported on Table II.

During the CS condition, participants tilted forwards, and twisted their upper body towards the standing leg (Fig. 6). They mainly used their right arm to initiate the movement by accelerating it forwards, and rotating it towards the standing leg around the vertical axis. The trunk rotated around the AP and ML axes towards the standing leg, which induced forwards and lateral accelerations in the same direction. The left arm showed less repeatable patterns. Acceleration phases were short and of low amplitude compared to the FS condition (see Table II), and were followed by a high-amplitude deceleration phase, as shown in Fig. 5. However, subjects showed higher angular velocities in both the AP and ML planes for all three IMUs.

\section{B. Offline classification of the Free Setting (FS) condition signals}

Table III shows the results from the LDA-based offline classification architecture on the FS condition signals, for both the intra-subject and inter-subject evaluations. In order to assess the classifier's ability to discriminate between all different classes, including LS and RS, it was run over the entire walk for each trial (EW data). However, classification 

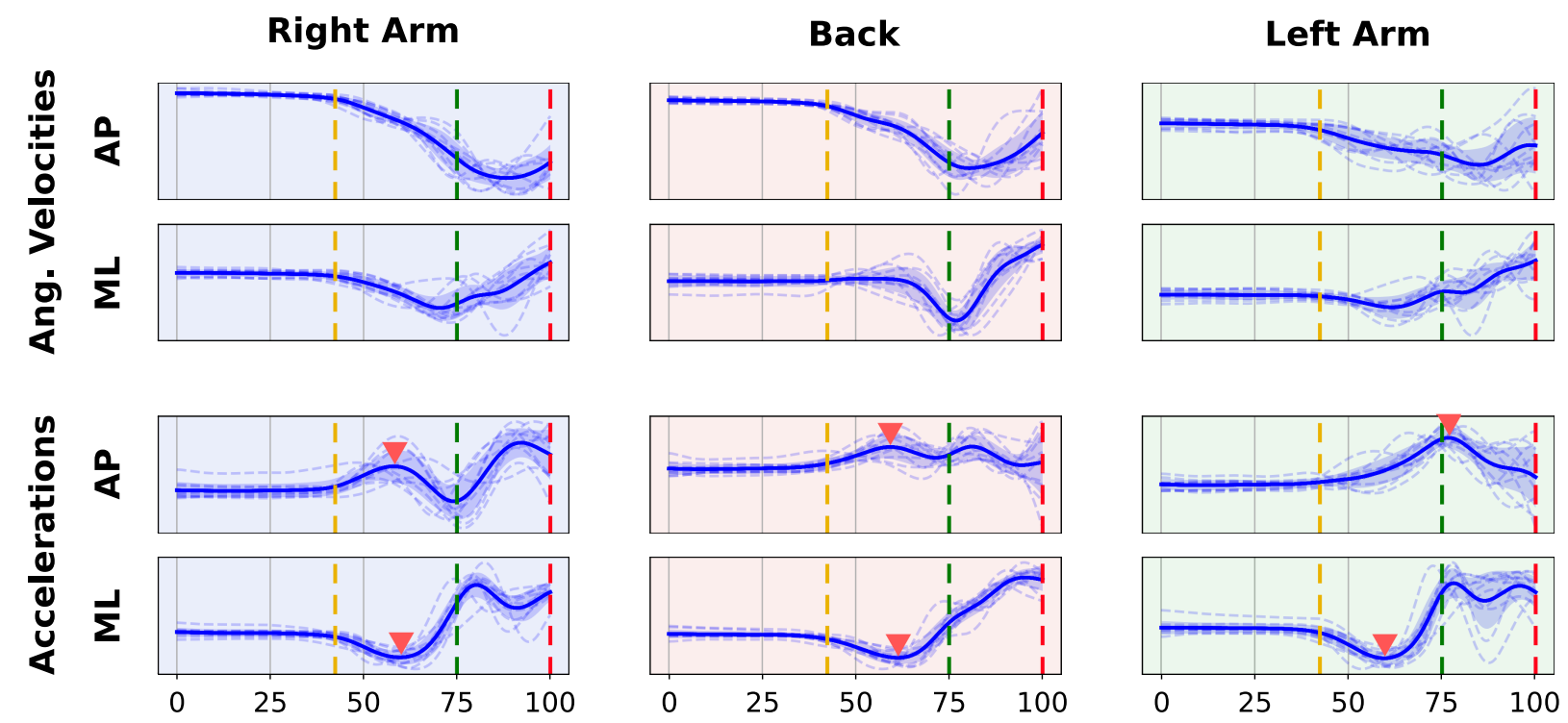

\section{- Heel-off \\ - Heel Strike \\ - Movement onset \\ $\checkmark$ Peak Acc}

Time Advancement [\%]

Fig. 3. Average accelerations and angular velocities recorded by all three IMUs during the FS condition trials in the ML and AP planes. The dashed lines represent the average signals for each subject, and the thick line corresponds to the overall average, with its corresponding standard deviation. Signals are represented from $0.5 \mathrm{~s}$ before the movement onset up to the heel-strike. Peak accelerations as defined in Table II are also indicated in the figure.
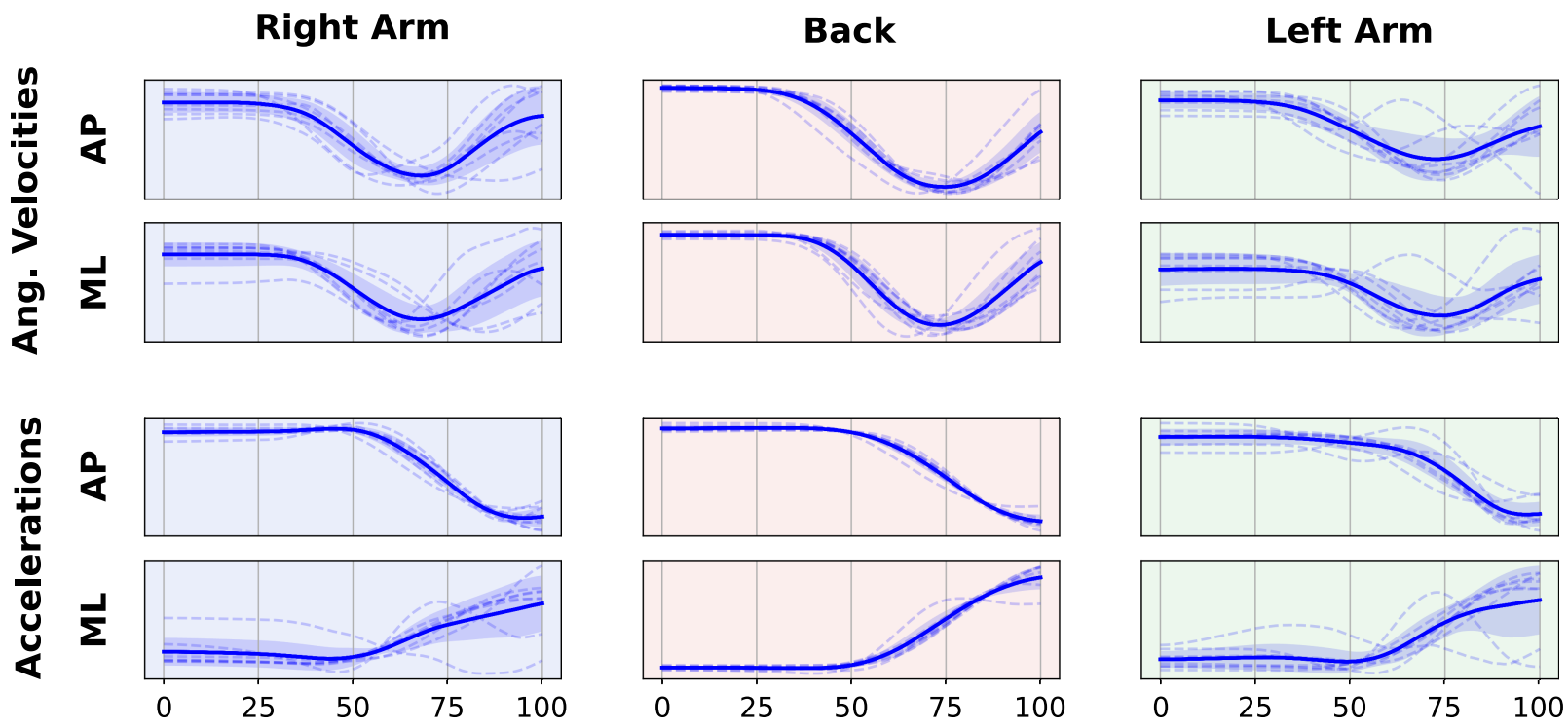

Time Advancement [\%]

Fig. 4. Average accelerations and angular velocities recorded by all three IMUs during the CS condition trials in the ML and AP planes. The dashed lines represent the average signals for each subject, and the thick line corresponds to the overall average, with its corresponding standard deviation. Signals are represented from $0.5 \mathrm{~s}$ before the movement onset up to the first zero-crossing of the angular velocity around the vertical axis (not shown here). 
TABLE II

PeAK AVERAge $( \pm$ SD) GII ACCELERATIONS AND ANGUlaR VELOCITIES IN THE ML AND AP PLANES FOR ALL IMU PLACEMENTS IN BOTH THE FS AND CS CONDITIONS.

\begin{tabular}{lllllr}
\hline & & \multicolumn{2}{l}{ Peak Accelerations [g] } & \multicolumn{2}{l}{ Peak Angular Velocities [deg/s] } \\
& & AP Plane & ML Plane & AP Plane & ML Plane \\
\hline \multirow{3}{*}{ FS Condition } & Trunk & $0.05 \pm 0.03$ & $0.05 \pm 0.02$ & $21.5 \pm 7.1$ & $9.5 \pm 6.5$ \\
& Right Arm & $0.05 \pm 0.03$ & $0.06 \pm 0.02$ & $23.1 \pm 8.8$ & $10.3 \pm 5.3$ \\
& Left Arm & $0.1 \pm 0.05$ & $0.06 \pm 0.02$ & $18.9 \pm 8.3$ & $6.2 \pm 5.2$ \\
\hline \multirow{3}{*}{ CS Condition } & Trunk & $0.01 \pm 0.01$ & $0.01 \pm 0.02$ & $30.7 \pm 12.7$ & $19.2 \pm 9.6$ \\
& Right Arm & $0.03 \pm 0.03$ & $0.04 \pm 0.04$ & $21.2 \pm 13.9$ & $15.1 \pm 6.8$ \\
& Left Arm & $0.03 \pm 0.03$ & $0.04 \pm 0.05$ & $17.8 \pm 11.0$ & $15.0 \pm 8.7$ \\
\hline
\end{tabular}

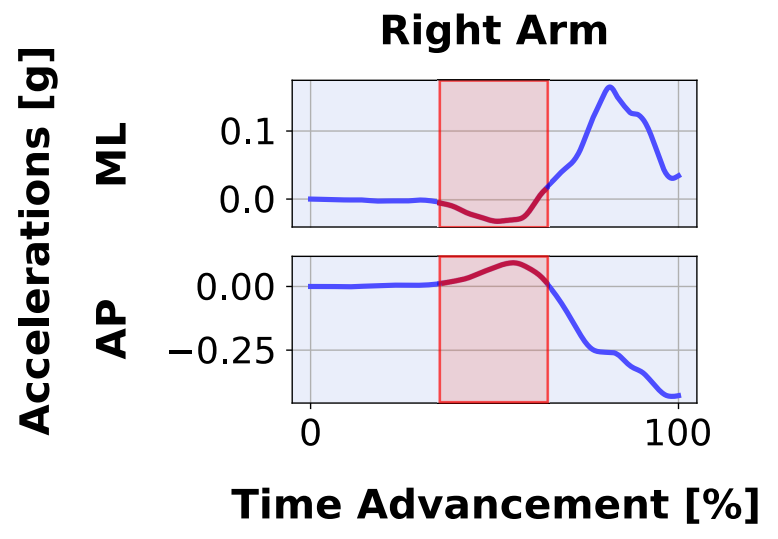

\section{Acceleration Phase}

Fig. 5. Typical AP acceleration pattern exhibited by one subject during one trial of the CS condition on the right arm IMU. The original signal is lowpass filtered by a 2nd order Butterworth filter with a $3 \mathrm{~Hz}$ cutoff frequency. The red zone indicates the precursor movements as defined in sec.III-A. The low-amplitude acceleration phase is distinguishable at the beginning of the movement, and is followed by a strong deceleration phase.

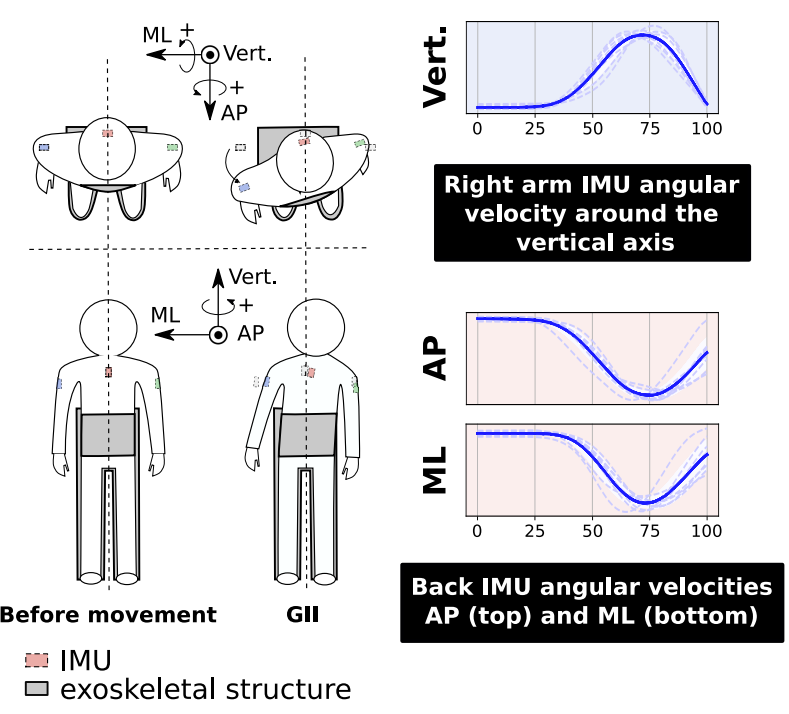

Fig. 6. General strategy exhibited by the participants during the CS condition to trigger the walking state of the robot.

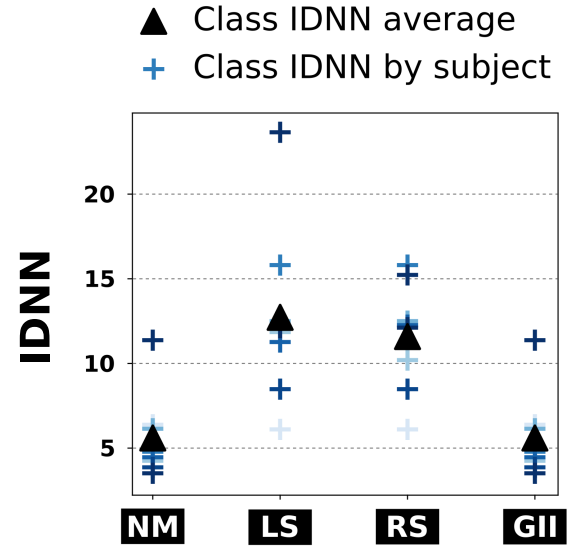

Fig. 7. The Inter-class Distance Nearest Neighbor (IDNN) metric computed in the original 198-dimensional feature space for each class and each subject for the intra classifiers. When the IDNN is the same for two classes, it means they are closest to each other.

accuracy over the first signal windows spanning from the beginning of each trial up to the end of the GII class was also reported in the Table (GII data). Overall, the average classification accuracy for the entire walk was $94.7 \%$, with a maximum accuracy value reaching $97.9 \%$ for the intra-subject setting, and $91.9 \%$ with a maximum value of $96.5 \%$ for the inter-subject setting. However, for all trials and all participants, gait intention was always correctly detected. Classification rates for the GII data were less than $100 \%$ because of time lags, meaning that the GII class was detected a few windows early or late in some cases. The average time lag for intrasubject classification was $0.17 \mathrm{~s}$, and $0.38 \mathrm{~s}$ for inter-subject classification, which explains the average loss of $2.5 \%$ in the classification rate between both classification schemes for the GII data.

\section{Online classification of the CS condition signals}

Fig. 7 represents the Inter-class Distance Nearest Neighbor (IDNN) metric for each subject.

Fig. 8 shows the subject-specific and overall results per classifier used from the real-time classification of IMU signals during the CS condition, for all eight participants included in the CS study. The "Other class detected first" label represents trials for which the Gait Initiation Intention (GII) class was 
TABLE III

RESULTS FROM THE OFFLINE CLASSIFICATION BASED ON THE FS CONDITION DATA

\begin{tabular}{lllllll}
\hline & $\begin{array}{l}\text { Classification rate } \\
\text { (EW data) }\end{array}$ & & \multicolumn{5}{l}{$\begin{array}{l}\text { Classification rate } \\
\text { (GII data) }\end{array}$} \\
\hline & average $( \pm$ SD) & $\max$ & $\min$ & average $( \pm$ SD) & $\max$ & $\min$ \\
\hline Intra-subject classification & $94.7 \%( \pm 1.5 \%)$ & $97.9 \%$ & $92.9 \%$ & $97.6 \%( \pm 1.0 \%)$ & $98.7 \%$ & $96.4 \%$ \\
Inter-subject classification & $91.9 \%( \pm 2.4 \%)$ & $96.5 \%$ & $87.8 \%$ & $95.1 \%( \pm 3.5 \%)$ & $98.3 \%$ & $89.0 \%$ \\
\hline
\end{tabular}

$\mathbf{A}$

$5153 \quad 5456 \quad 57 \quad 5859510$

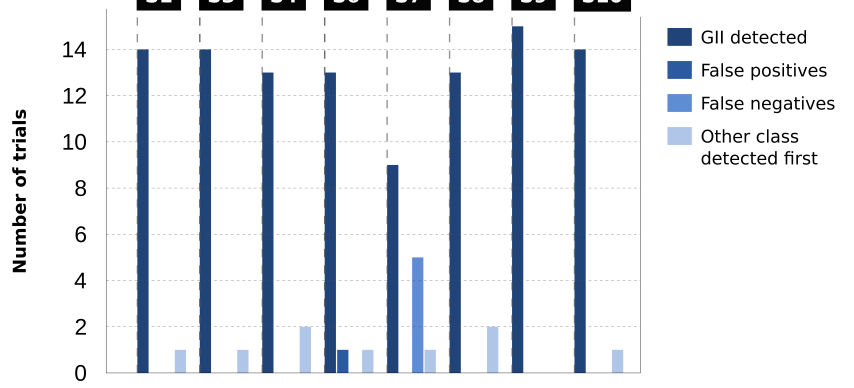

B

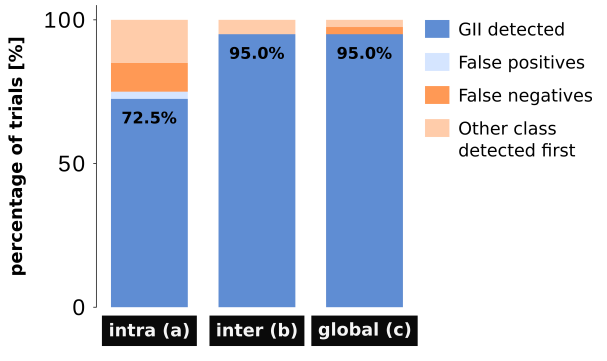

Fig. 8. (A) Subject-specific results from the CS condition. (B) Overall results from the real-time classification during the CS condition per classifier used

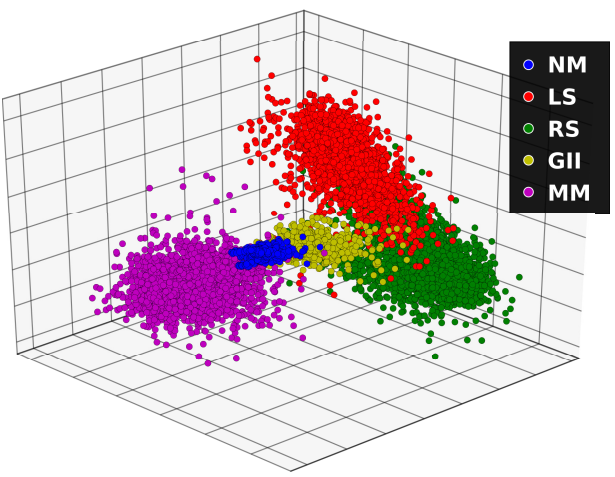

Fig. 9. Representation of the three-dimensional LDA-generated projection subspace based on FS training data from all subjects (global classifier), with additional MM data recorded from eight subjects.

detected after another class was detected first. These trials were considered as false negatives, even if detection of gait intention occurred afterwards. The only false positive detection occurred during one intra trial (Subject 6 in Fig. 8-A). 64.3\% of false negatives were due to another class being detected first, and the remaining ones all came from the same subject (Subject 7 in Fig. 8-A). Overall, the robot's walk was correctly triggered in respectively $72.5 \%$ of the intra (a) trials, $95 \%$ of the inter (b) trials, and $95 \%$ of the global (c) trials.

A Kruskall-Wallis test showed a statistically significant difference between the three classifiers $(H=6.35, p=0.042)$. An additional post-hoc Dunn test showed that there was no statistical difference in the performance results between the inter and global classifiers $(p=1.00)$. However, performance of both classifiers were statistically different from that of the intra classifier ( $p=0.029$ for both comparisons).

On average, the GII class was detected $514 \mathrm{~ms}( \pm 373 \mathrm{~ms}$ $\mathrm{SD})$ after the movement onset, which approximately corresponds to the duration of one buffer window $(500 \mathrm{~ms})$, and falls within the range of Anticipatory Postural Adjustments (APAs) duration.

\section{Testing the classifier for false Gait Initiation Intention} (GII) positives induced by everyday movements

Testing the global classifier on the Miscellaneous Movements (MM) recordings showed that on average, $27.6 \%$ of the performed movements windows were classified as GII, and only $7.2 \%$ as either Left Step (LS) or Right Step (RS). $64.6 \%$ of the windows were classified as No Movement (NM), because of transition resting periods, and because the performed movements implied that either one of the arms could be at rest - meaning that data points were likely to maximize the distance to the NM hyperplane in the LDA features-based subspace.

By adding the MM label to the global classifier training data set using half of the MM recordings, and using the other half as a testing set, there was an average of $98.5 \%$ windows classified as either MM or NM, with $1.5 \%$ windows falsely classified as GII. Fig. 9 shows a three-dimensional LDAgenerated projection subspace of this 5-class classification problem. As can be seen on the figure, the five classes form highly discriminated clusters of points with little overlap, and the MM and GII class are well separated

Additionally, the MM-enriched classifier was tested offline on the trials from the CS condition corresponding to the global classifier (c) for the eight participants included in the CS study. This showed that the GII class was consistently detected in all trials but one. The remaining trial was a False Negative where the MM class was detected instead.

\section{DISCUSSION}

\section{A. Qualitative analysis of upper-body kinematic signals}

1) Free Setting (FS): Results for the trunk IMU in the FS condition are consistent with previous studies [24], [26], 
[27], showing that the upper body is accelerated forwards, and towards the standing leg. Therefore, it was possible to validate our method to evaluate kinematic patterns that anticipate gait initiation based on inertial sensors placed on the upper body.

To our knowledge, there is no work investigating arm kinematics during Anticipatory Postural Adjustments (APAs), but shoulder movements have been studied before [24]. Our results suggest that both arms follow the same acceleration patterns as the trunk, and that the arms also exhibit repeatable precursor patterns before the heel-off. The overall movement is initiated by the side ipsilateral to the stepping leg (the right side in this study), since the contralateral arm exhibits a delay in its forward acceleration, with a lower peak angular velocity in the Antero-Posterior (AP) plane. Therefore, the ipsilateral side of the upper body starts to shift forwards and towards the standing leg, and the contralateral arm follows the general forwards movement before the heel leaves the ground. Additionally, standard deviations for average acceleration peak values reported in Table II confirm that the amplitudes of arms and trunk movements are highly variable between subjects.

2) Constrained Setting (CS): During the CS condition, the participants focused on using the side of their upper body ipsilateral to the stepping leg (the right side) to initiate gait. Since their legs were constrained by the exoskeleton, they seemed to focus on upper body movements that they imagined would help lifting the robot's leg, and pushing it forwards. This was achieved by rotating the upper body laterally, and tilting it forwards, while strongly bringing their right shoulder towards the standing leg. Contrary to the FS condition, where precursor patterns are unconscious, they actively engaged in using the right side of their upper body to initiate gait, and exhibited higher angular velocities in the Medio-Lateral (ML) and Antero-Posterior (AP) planes for the trunk IMU. The phase of forwards acceleration was short and of low amplitude compared to the FS condition, and was followed by a high amplitude deceleration. This shows that all participants relied on a similar movement strategy that actively focused on using the ipsilateral side of their upper body to bring the robot to start moving, and intuitively used their right arm in a similar way that they naturally did during the FS condition, with forwards accelerations that lasted for shorter duration. The left arm exhibited less repeatable movements that were mainly due to the dynamics of the right side, and to whether participants controlled their arm or not. Similarly to the FS condition, standard deviations for average acceleration peak values reported in Table II confirm that the amplitudes of arms and trunk movements are highly variable between subjects in the CS condition as well.

\section{B. Evaluation of Gait Initiation detection in the Free Setting (FS) based on an offline classification architecture}

1) Intra-subject evaluation: Evaluation of the intra-subject classifiers on the Entire Walk data from the FS condition returned accuracy scores higher than 94\%, showing that the LDA architecture is capable of correctly discriminating between the different labeled classes in a subject-specific setting. Additionally, loss of accuracy is mainly due to time lags (either positive or negative) during transitions between different classes. Since the Gait Initiation Intention (GII) class is consistently and correctly detected for all trials, this evaluation also confirms that subject-specific kinematic patterns that precede gait initiation are repeatable in the FS condition, and that intra-subject classifiers based on FS data could be used in the CS condition to check for similarities in these patterns between both conditions.

2) Inter-subject evaluation: Evaluation of the inter-subject classifiers on the Entire Walk data from the FS condition returned accuracy scores higher than $91 \%$, showing that the LDA architecture is also capable of correctly discriminating between the different labeled classes based on data acquired from a pool of subjects that does not contain the tested participant's trials. However, loss of accuracy is higher on average than the intra-subject evaluation, since time lags can be longer. Again, the GII class is consistently and correctly detected for all trials, which shows that the variability of kinematic patterns that precede gait initiation between participants in the FS condition is low, and that inter-subject classifiers based on FS data could be used in the CS condition as well. This consistency is also supported by Fig 10 in the appendix, which illustrates the two-dimensional projections of the intraclassifiers. The Inter-class Distance Nearest Neighbor (IDNN) metric in Fig. 7 additionally confirms that the No Movement (NM) and GII clusters are consistently closest to each other, and that both step classes are closest to each other on average. This supports the fact that anticipatory patterns preceding gait initiation exhibit low-amplitude dynamics that are separable from walking-related movements.

\section{Experimental evaluation of gait initiation detection in the exoskeleton based on a free setting training set}

Based on our experimental results, the LDA architecture could be successfully used to detect gait initiation intention for a majority of the CS trials, even though subjects exhibited upper body patterns that did not correspond exactly with those of the FS condition, which provided the training data.

One of the main differences between both conditions lied in the signal amplitudes and duration of the acceleration phases. Additionally, the left arm IMU did not exhibit repeatable patterns. However, it is hypothesized that the amplitude ranges remained closer to what can be exhibited by the trained GII class, meaning that in the feature-based subspace formed by the LDA architecture, data points extracted from the participants' movements in the CS condition fell in the GII class region. This is illustrated in Fig 11 in the appendix. Additionally, the IDNN metric was calculated between the clusters formed by the data points classified as GII in the CS condition and the four labeled classes for the intra-classifier trials where Gait Initiation was correctly detected (see Table V in the appendix). In 6 subjects out of 8, the IDNN for the data points classified as GII in the CS condition was equal to the distance to the GII class, showing that the testing points were on average closest to the GII cluster. In the remaining two subjects, the testing clusters were closest to the Left Step class, supporting the fact that the anticipatory 
patterns preceding gait initiation exhibited stronger dynamics when the participants were equipped with the exoskeleton. However, this did not affect the LDA output, which correctly classified the testing points as GII, since the IDNN relies on the Mahalanobis distance, which introduces a distortion of the feature space through covariance-based normalization, while the LDA classifies new data points by computing the signed euclidean distances to the class hyperplanes.

Alternatively, these results show that the constructed classifier might not be discriminative enough so that the GII class would actually be specific to gait initiation precursor patterns, and that similar low-amplitude movements, such as handshakes or reaching movements, could also be detected as GII. Indeed, both the FS and CS conditions were conducted in a controlled setting, where the participants were asked not to move before the audio cue was heard. However, this is not representative of the real-life use of an assistive lowerlimb exoskeleton, in which the users would freely move their upper body while the robot is standing still in an upright position. In the classification architecture that was developed for this experimental work, such movements could possibly lead to false GII positives, and a preliminary experiment was conducted in order to assess the robustness of the classifier to such events.

\section{Experimental evaluation of the robustness of the global classifier to false Gait Initiation Intention (GII) positives}

Testing the global classifier on the recordings from the False Positives (FP) experiment showed that Miscellaneous Movements (MM) were prone to be classified as Gait Initiation Intentions, rather than one of the other movement classes (Left Step or Right Step). This shows that Miscellaneous Movements exhibit features that are similar to the GII class, and can induce false GII positives if not taken into account when training the classifiers. This is not a desired behaviour during the normal use of a lower-limb assistive exoskeleton. However, when adding the FP recordings to the training set of the global classifier, GII false positives were reduced to only $1.5 \%$, most of which happened punctually (meaning that one window was classified as GII in between correctly-classified MM windows). This rate could be further reduced by adding a filter which would not initiate gait of the robot in such cases. Fig. 9 also shows that the classified movements are differentiable, and confirms the specificity of the patterns used to detect gait initiation intention. Additionally testing the 5class classifier on the CS condition confirms that the enriched classification architecture can be used to effectively prevent false GII positives, with limited false negative rates, and no loss of performance compared to the original global classifier.

\section{E. Limitations and prospects of the study}

This study shows that anticipatory movements of the upperbody before gait initiation seem to be retrieved in able-bodied participants using a lower-limb exoskeleton. The participants exhibited a similar movement strategy to trigger gait in the robot. However, further experiments need to be conducted with more subjects to refine and generalize these results, and strengthen the statistical analysis. For example, by involving more participants who have no previous experience in using the exoskeleton, it would be possible to assess the influence of usage experience on both the movement strategies, and the classification outcome. More trials could also be necessary to assess how strategies can evolve in time, and whether participants are able to adapt to false classification negative occurrences by performing sets of movements that are even more specific, and with less variability.

Additional feature selection steps should also be considered to further reduce the high number of dimensions of the feature space, and properly avoid the overfitting that can occur in more generic settings. This study systematically used all 18 IMU signals to construct the LDA classifiers. However, signal-specific selection can be performed, and the possible redundancies between both arm IMUs due to their symmetrical placement can be exploited.

It is also important to note that lower-limb assistive devices are aimed at being used with SCI patients with different injury levels. Therefore, it is necessary to evaluate how these various conditions can affect the upper-body movements. The Atalante exoskeleton was specifically designed to be used by paraplegic patients who still have mobility above the waist level. It is hypothesized that such a category of patients could rely on similar movement strategies to express gait initiation intention than the able-bodied participants included in this study. Further experiments with paraplegic patients need to be conducted to confirm this hypothesis. If it is not confirmed, the classification architecture could still be used by asking the patients to perform any movements they find intuitive to trigger the walking state of the robot. Recording of such movements can then be used as a basis to construct a training data set for a new patient-specific classifier.

\section{CONCLUSION}

This work showed that subjects in a non-back drivable exoskeleton actively engaged in a gait initiation strategy similar to the natural precursor patterns exhibited in a free environment, by shifting the right side of their upper body forwards and towards the standing leg. By building a standard classification architecture using free walking data as a training set, gait initiation intention was then successfully detected when starting from an upright standstill position in the lowerlimb assistive exoskeleton. Robustness of the classifier against false positives triggered by everyday movements was assessed in a supplementary experiment by adding real life gestures to the training set, which confirmed that the classification architecture could be made more robust to false gait initiation positives.

Further experiments need to be conducted for refinement and generalization of the results. Paraplegic patients can then be included in more ecological environments to assess the transferability of these methods and the able-based classifiers to more realistic mobility scenarios and real-life use cases. However, this work is encouraging for future developments for machine-learning-based control strategies in lower limb assistive exoskeletons. 
S1

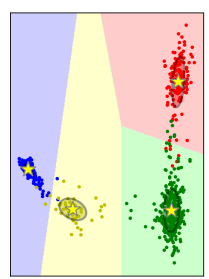

56

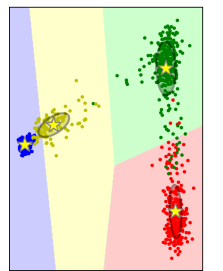

S2

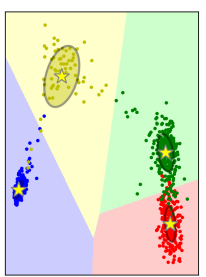

S7

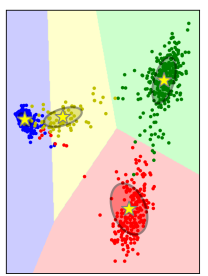

S3

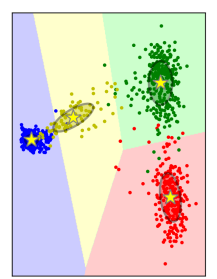

58

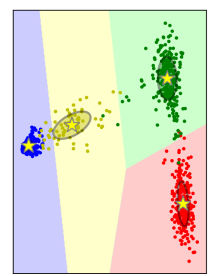

54

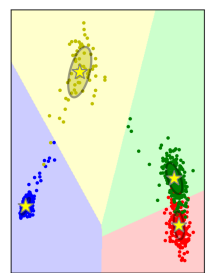

S9

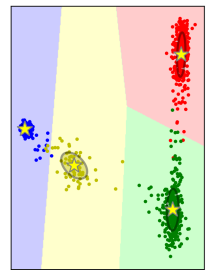

S5

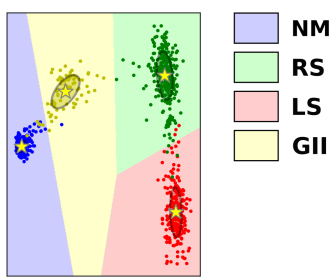

S10

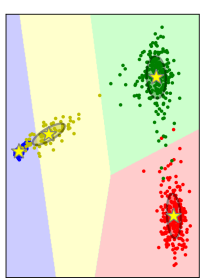

Fig. 10. Representation of the intra classifiers in the two-dimensional LDA-generated projection subspace. The different colored regions represent the classifier decision regions for each of the four labeled classes (NM, RS, LS, GII).

TABLE IV

DistANCE METRIC $m_{i, j}$ COMPUTED FOR ALL CLASSES AND ALL PARTICIPANTS. BOLD VALUES REPRESENT THE IDNN FOR EACH CLASS AND EACH PATIENT.

\begin{tabular}{llllllllllll}
\hline Class i & Class j & S1 & S2 & S3 & S4 & S5 & S6 & S7 & S8 & S9 & S10 \\
\hline NM & LS & 33.77 & 126.92 & 30.43 & 207.13 & 107.67 & 61.31 & 10.03 & 98.48 & 94.9 & 98.65 \\
& RS & 26.1 & 38.57 & 17.12 & 45.71 & 33.87 & 29.65 & 33.38 & 36.6 & 53.89 & 26.7 \\
& GII & $\mathbf{6 . 3 5}$ & $\mathbf{4 . 3}$ & $\mathbf{4 . 2 2}$ & $\mathbf{6 . 1 1}$ & $\mathbf{6 . 2}$ & $\mathbf{4 . 7 5}$ & $\mathbf{4 . 4 3}$ & $\mathbf{3 . 8 3}$ & $\mathbf{1 1 . 3 4}$ & $\mathbf{3 . 4 8}$ \\
\hline LS & NM & 33.77 & 126.92 & 30.43 & 207.13 & 107.67 & 61.31 & $\mathbf{1 0 . 0 3}$ & 98.48 & 94.9 & 98.65 \\
& RS & $\mathbf{6 . 9 1}$ & $\mathbf{7 . 2 3}$ & $\mathbf{1 1 . 8 1}$ & $\mathbf{1 7 . 2 9}$ & $\mathbf{1 4 . 7 9}$ & $\mathbf{1 5 . 8 5}$ & 14.22 & $\mathbf{8 . 4 1}$ & $\mathbf{1 1 . 9 2}$ & $\mathbf{2 0 . 1 2}$ \\
& GII & 15.96 & 22.3 & 20.58 & 30.39 & 37.58 & 34.77 & 14.15 & 26.87 & 38.87 & 42.1 \\
\hline RS & NM & 26.1 & 38.57 & 17.12 & 45.71 & 33.87 & 29.65 & 33.38 & 36.6 & 53.89 & 26.7 \\
& LS & $\mathbf{6 . 9 1}$ & $\mathbf{7 . 2 3}$ & $\mathbf{1 1 . 8 1}$ & $\mathbf{1 7 . 2 9}$ & 14.79 & 15.85 & 14.22 & $\mathbf{8 . 4 1}$ & $\mathbf{1 1 . 9 2}$ & 20.12 \\
& GII & 9.18 & 13.23 & 10.23 & 17.83 & $\mathbf{1 4 . 4 8}$ & $\mathbf{1 4 . 7 8}$ & $\mathbf{1 3 . 6 8}$ & 12.7 & 17.83 & $\mathbf{1 5 . 1 1}$ \\
\hline GII & NM & $\mathbf{6 . 3 5}$ & $\mathbf{4 . 3}$ & $\mathbf{4 . 2 2}$ & $\mathbf{6 . 1 1}$ & $\mathbf{6 . 2}$ & $\mathbf{4 . 7 5}$ & $\mathbf{4 . 4 3}$ & $\mathbf{3 . 8 3}$ & $\mathbf{1 1 . 3 4}$ & $\mathbf{3 . 4 8}$ \\
& LS & 15.96 & 22.3 & 20.58 & 30.39 & 37.58 & 34.77 & 14.15 & 26.87 & 38.87 & 42.1 \\
& RS & 9.18 & 13.23 & 10.23 & 17.83 & 14.48 & 14.78 & 13.68 & 12.7 & 17.83 & 15.11 \\
\hline
\end{tabular}

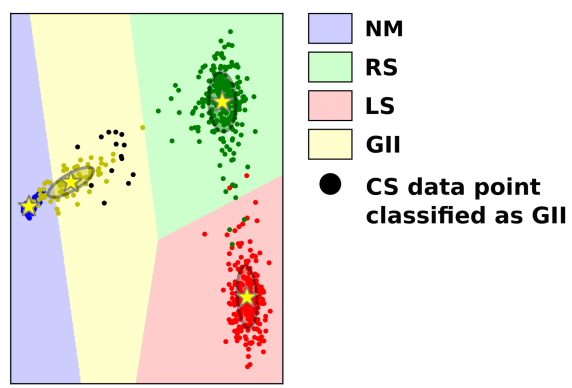

Fig. 11. Representation of the LDA-generated projection subspace based on FS data training from one subject. The black points represent projected features from data windows which have been classified as GII from CS trials from the same subject. These data points, though far from the GII class centroid generated by the training data, are still closer to it than the other classes.

\section{APPENDIX A}

COMPutation of the InTER-Class Distance Nearest NEIGHBOR (IDNN)

The IDNN for class $i$ is defined as in [31]:
TABLE V

DISTANCE METRICS USED FOR THE COMPUTATION OF THE IDNN BETWEEN THE TESTING POINTS CLUSTERS AND THE FOUR CLASS CLUSTERS. THE BOLD VALUES INDICATE THE IDNN VALUE FOR EACH SUBJECT.

\begin{tabular}{lllllllll}
\hline & S1 & S2 & S3 & S4 & S5 & S6 & S7 & S8 \\
\hline NM & 4.99 & 3.56 & 2.56 & 2.73 & 4.01 & 3.76 & 3.96 & 4.26 \\
RS & 5.44 & 4.13 & 2.06 & $\mathbf{1 . 6 1}$ & $\mathbf{2 . 5 2}$ & 6.60 & 5.14 & 6.47 \\
LS & 4.94 & 3.18 & 1.80 & 2.09 & 2.80 & 5.06 & 5.58 & 5.00 \\
GII & $\mathbf{3 . 4 7}$ & $\mathbf{2 . 3 2}$ & $\mathbf{1 . 0 6}$ & 2.12 & 3.71 & $\mathbf{0 . 9 3}$ & $\mathbf{3 . 8 2}$ & $\mathbf{2 . 0 5}$ \\
\hline
\end{tabular}

$$
I D N N_{i}=\min _{j \in C, j \neq i} \underbrace{\frac{d_{j}^{i} \times d_{i}^{j}}{d_{j}^{i}+d_{i}^{j}}}_{m_{i, j}}
$$

where $C=\{1,2,3,4\}$ is the set of labeled classes, and $d_{j}^{i}$ is the Mahalanobis distance from the cluster of class $j$ to the cluster of class $i$, given the covariance matrix $\mathbf{S}_{\mathbf{i}}$ of cluster $i$, and the centroid coordinates $\mu_{i}$ and $\mu_{j}$ of clusters $i$ and $j$ :

$$
d_{j}^{i}=\frac{1}{2} \sqrt{\left(\mu_{i}-\mu_{j}\right)^{T} \mathbf{S}_{\mathbf{i}}^{-1}\left(\mu_{i}-\mu_{j}\right)}
$$


Table IV shows the distance metrics $m_{i, j}$ used for the computation of the IDNN for all labeled classes and all subjects in the intra-classifiers, as shown by Fig. 7 in III-C. Table $\mathrm{V}$ shows the distance metrics used for the computation of the IDNN between the testing points clusters and the four class cluster in IV-C.

\section{APPENDIX B}

\section{REPRESENTATION OF THE LDA GENERATED SUBSPACES}

Fig. 10 shows the structure of the individual intra-classifiers constructed from the Free Setting training data as projected on the LDA-computed two-dimensional space. For all subjects, the four class clusters seem well separated and organized in the same fashion, with the GII cluster being closer to the NM cluster, and appearing as a transition between the resting state and the two walking states (Left and Right). This supports the consistency with which the classifiers are able to discriminate between the different classes.

Fig. 11 illustrates how data windows classified as Gait Initiation Intentions in the Constrained Setting can fall in the GII region, but far from the class centroid.

\section{ACKNOWLEDGEMENT}

The study was financially supported by the Wandercraft company and the French state funds through the CIFRE program managed by the ANRT (Association Nationale Recherche Technologie), under reference $N^{\circ} 2018 / 0378$.

\section{REFERENCES}

[1] S. Viteckova, P. Kutilek, and M. Jirina, "Wearable lower limb robotics: A review," Integrative Medicine Research, vol. 33, pp. 96-105, 2013.

[2] T. Yan, M. Cempini, C. M. Oddo, and N. Vitiello, "Review of assistive strategies in powered lower-limb orthoses and exoskeletons," Robotics and Autonomous Systems, vol. 64, pp. 120-136, 2015.

[3] B. Chen, H. Ma, L.-Y. Qin, F. Gao, K.-M. Chan, S.-W. Law, L. Qin, and W.-H. Liao, "Recent developments and challenges of lower extremity exoskeletons," Journal of Orthopaedic Translation, vol. 5, pp. 26-37, 2016.

[4] R. Riener, "The Cybathlon promotes the development of assistive technology for people with physical disabilities," Journal of NeuroEngineering and Rehabilitation, vol. 13, no. 1, p. 49, 2016.

[5] W. Huo, S. Mohammed, J. C. Moreno, and Y. Amirat, "Lower Limb Wearable Robots for Assistance and Rehabilitation: A State of the Art," IEEE Systems Journal, vol. 10, no. 3, pp. 1068-1081, 2016.

[6] A. Esquenazi, M. Talaty, A. Packel, and M. Saulino, "The Rewalk powered exoskeleton to restore ambulatory function to individuals with thoracic-level motor-complete spinal cord injury," American Journal of Physical Medicine and Rehabilitation, vol. 91, no. 11, pp. 911-921, 2012.

[7] G. Barbareschi, R. Richards, M. Thornton, T. Carlson, and C. Holloway, "Statically vs dynamically balanced gait: Analysis of a robotic exoskeleton compared with a human," Proceedings of the Annual International Conference of the IEEE Engineering in Medicine and Biology Society, EMBS, vol. 2015-Novem, pp. 6728-6731, 2015.

[8] T. Gurriet, S. Finet, G. Boeris, A. Duburcq, A. Hereid, O. Harib, M. Masselin, J. Grizzle, and A. D. Ames, "Towards Restoring Locomotion for Paraplegics: Realizing Dynamically Stable Walking on Exoskeletons," in 2018 IEEE International Conference on Robotics and Automation (ICRA). IEEE, may 2018, pp. 2804-2811.

[9] O. Harib, A. Hereid, A. Agrawal, T. Gurriet, S. Finet, G. Boeris, A. Duburcq, M. E. Mungai, M. Masselin, A. D. Ames, K. Sreenath, and J. W. Grizzle, "Feedback Control of an Exoskeleton for Paraplegics: Toward Robustly Stable, Hands-Free Dynamic Walking," IEEE Control Systems, vol. 38, no. 6, pp. 61-87, dec 2018.

[10] W. Seymour, Remaking the Body. Routledge, feb 1998.

[11] M. Casadio, R. Ranganathan, and F. A. Mussa-Ivaldi, "The BodyMachine Interface: A new perspective on an old theme," Journal of Motor Behaviour, vol. 44, no. 6, pp. 419-433, 2012.
[12] J. Lobo-Prat, A. Q. Keemink, A. H. Stienen, A. C. Schouten, P. H. Veltink, and B. F. Koopman, "Evaluation of EMG, force and joystick as control interfaces for active arm supports."

[13] A. L. Benabid, T. Costecalde, A. Eliseyev, G. Charvet, A. Verney, S. Karakas, M. Foerster, C. Mestais, and S. Chabardes, "Articles An exoskeleton controlled by an epidural wireless brain - machine interface in a tetraplegic patient : a proof-of-concept demonstration," vol. 4422, no. 19, pp. 1-11, 2019.

[14] D. Xu, X. Liu, and Q. Wang, "Knee Exoskeleton Assistive Torque Control Based on Real-Time Gait Event Detection," IEEE Transactions on Medical Robotics and Bionics, vol. 1, no. 3, pp. 158-168, 2019.

[15] C. Castellini, P. Artemiadis, M. Wininger, A. Ajoudani, M. Alimusaj, A. Bicchi, B. Caputo, W. Craelius, S. Dosen, K. Englehart, D. Farina, A. Gijsberts, S. B. Godfrey, L. Hargrove, M. Ison, T. Kuiken, M. Markoví, P. M. Pilarski, R. Rupp, E. Scheme, R. Chavarriaga, and S. Ramamoorthy, "Proceedings of the first workshop on Peripheral Machine Interfaces: going beyond traditional surface electromyography," 2014.

[16] J. Wolpaw and E. W. Wolpaw, Brain-computer interfaces: principles and practice. OUP USA, 2012.

[17] H. L. Bartlett and M. Goldfarb, "A Phase Variable Approach for IMU-Based Locomotion Activity Recognition," IEEE Transactions on Biomedical Engineering, vol. 65, no. 6, pp. 1-1, 2017.

[18] L. Bao and S. S. Intille, "Activity Recognition from User-Annotated Acceleration Data," Pervasive, pp. 1-17, 2004.

[19] D. M. Karantonis, M. R. Narayanan, M. Mathie, N. H. Lovell, and B. G. Celler, "Implementation of a real-time human movement classifier using a triaxial accelerometer for ambulatory monitoring," IEEE Transactions on Information Technology in Biomedicine, vol. 10, no. 1, pp. 156-167, 2006.

[20] S. J. Preece, J. Y. Goulermas, L. P. J. Kenney, D. Howard, K. Meijer, and R. Crompton, "Activity identification using body-mounted sensors-a review of classification techniques," Physiological Measurement, vol. 30, pp. 1-33, 2009.

[21] I. Farkhatdinov, N. Roehri, and E. Burdet, "Anticipatory detection of turning in humans for intuitive control of robotic mobility assistance," Bioinspiration \& Biomimetics, vol. 12, no. 5, 2017.

[22] C. Pelachaud, "Head, Shoulders and Hips Behaviors during Turning," in Lecture Notes in Computer Science, 2013, vol. 8212, no. January 2014.

[23] D. Novak, M. Goršič, J. Podobnik, and M. Munih, "Toward Real-Time Automated Detection of Turns during Gait Using Wearable Inertial Measurement Units," Sensors, vol. 14, no. 10, pp. 18 800-18 822, oct 2014.

[24] Y. Brenière and G. Dietrich, "Heel-off perturbation during gait initiation: biomechanical analysis using triaxial accelerometry and a force plate.' Journal of biomechanics, vol. 25, no. 2, pp. 121-7, feb 1992.

[25] M. Mancini, C. Zampieri, P. Carlson-Kuhta, L. Chiari, and F. B. Horak, "Anticipatory postural adjustments prior to step initiation are hypometric in untreated Parkinson's disease: An accelerometer-based approach," European Journal of Neurology, vol. 16, no. 9, pp. 1028-1034, 2009.

[26] M. Mancini, L. Chiari, L. Holmstrom, A. Salarian, and F. B. Horak, "Validity and reliability of an IMU-based method to detect APAs prior to gait initiation," Gait and Posture, vol. 43, pp. 125-131, 2016.

[27] R. Martinez-Mendez, M. Sekine, and T. Tamura, "Detection of anticipatory postural adjustments prior to gait initiation using inertial wearable sensors," Journal of NeuroEngineering and Rehabilitation, vol. 8, no. 1, p. 17, apr 2011.

[28] D. Novak, P. Reberšek, S. M. M. De Rossi, M. Donati, J. Podobnik, T. Beravs, T. Lenzi, N. Vitiello, M. C. Carrozza, and M. Munih, "Automated detection of gait initiation and termination using wearable sensors," Medical Engineering \& Physics, vol. 35, no. 12, pp. 17131720, dec 2013

[29] T. Hastie, R. Tibshirani, and J. Friedman, The Elements of Statistical Learning, ser. Springer Series in Statistics. New York, NY, USA: Springer New York Inc., 2001.

[30] S. J. Preece, J. Y. Goulermas, L. P. Kenney, and D. Howard, "A comparison of feature extraction methods for the classification of dynamic activities from accelerometer data," IEEE Trans Biomed Eng, vol. 56, no. 3, pp. 871-879, 2009.

[31] M. B. Kristoffersen, A. W. Franzke, C. K. van der Sluis, A. Murgia, and R. M. Bongers, "The Effect of Feedback During Training Sessions on Learning Pattern-Recognition-Based Prosthesis Control,' IEEE Transactions on Neural Systems and Rehabilitation Engineering, vol. 27, no. 10, pp. 2087-2096, oct 2019. 